Somnologie $2018 \cdot 22: 1$

https://doi.org/10.1007/s11818-018-0155-7

(c) Springer Medizin Verlag GmbH, ein Teil von Springer Nature 2018

CrossMark

Kai Spiegelhalder

Klinik für Psychiatrie und Psychotherapie, Universitätsklinikum Freiburg, Freiburg, Deutschland

\title{
Psychologische Schlafforschung
}

Die Psychologie ist die empirische Wissenschaft vom menschlichen Erleben und Verhalten. Psychologische Methoden sind dadurch auch in der Schlafforschung relevant, z. B. bei der Erfassung der subjektiven Wahrnehmung des Schlafs, bei der Untersuchung von psychologischen Ursachen von Schlafstörungen oder bei Anwendung klinisch-psychologischer Interventionen zur Verbesserung des Schlafs. Das Schwerpunktthema der vorliegenden Ausgabe der Somnologie ist die psychologische Schlafforschung, wobei sich mehrere Beiträge der psychologischen Sichtweise auf den gesunden und gestörten Schlaf widmen.

So untersuchten Dr. Gieselmann und Kollegen die Rolle von Selbststeuerungsfähigkeiten für die Schlafqualität. Dabei zeigte sich, dass der bereits bekannte Zusammenhang zwischen Neurotizismus und Schlafqualität nur für solche Menschen vorhanden ist, die ausgeprägt lageorientiert sind, was bedeutet, dass es diesen Menschen schwerfällt, sich von Gedanken und Gefühlen zu lösen, um anstehende Aufgaben zu erledigen. Weiterhin finden Sie in dieser Ausgabe einen Artikel von Prof. Schlarb und Kollegen zu den Zusammenhängen des Schlafs mit arbeitsbezogenen Variablen, insbesondere mit Burn-out am Arbeitsplatz. Erwartungsgemäß zeigte sich hierbei, dass Arbeitnehmer, die sich am Arbeitsplatz überfordert fühlen, auch einen schlechteren Schlaf aufweisen, was die große Bedeutung des Arbeitslebens für unseren Schlaf unterstreicht. In einer weiteren Arbeit untersuchte Dr. Kelmanson den Schlaf von Vorschulkindern mit infantiler Zerebralparese. In Übereinstimmung mit der Hypothese der Studie fand sich hierbei eine Beeinträchtigung des Schlafs der betroffenen Kinder, die unter anderem mit der Ängstlichkeit der
Kinder zusammenhing. Darüber hinaus legten Herr Dieck und Kollegen eine deutsche Übersetzung des derzeit am häufigsten verwendeten Fragebogens im Bereich der Insomnieforschung vor, des Insomnia Severity Index. Dabei zeigen sich gute psychometrische Eigenschaften des übersetzten Fragebogens. Im letzten Beitrag zum Schwerpunktthema berichten Prof. Danker-Hopfe und Kollegen, welche aktuellen Studien in Zusammenarbeit mit der Gruppe Angewandte Militärpsychologische Forschung des Streitkräfteamts der Bundeswehr initiiert wurden. Dabei wird insbesondere ein Schlaf-CoachingProgramm evaluiert, das mit dem Ziel der Prävention bei Soldaten der Bundeswehr eingesetzt wird.

Ich hoffe, dass die vorliegende Ausgabe der Somnologie für Sie interessante Artikel beinhaltet und wünsche Ihnen eine anregende Lektüre.

Ihr

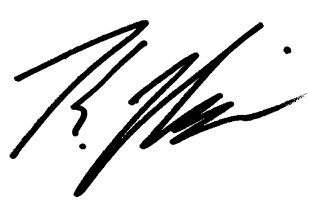

PD Dr. Dr. Kai Spiegelhalder

\section{Korrespondenzadresse}

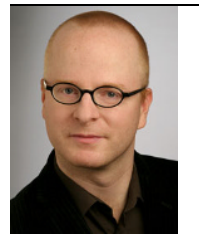

PD Dr. Dr. K. Spiegelhalder Klinik für Psychiatrie und Psychotherapie, Universitätsklinikum Freiburg Hauptstraße 5, 79104 Freiburg, Deutschland Kai.Spiegelhalder@uniklinikfreiburg.de

Interessenkonflikt. K. Spiegelhalder gibt an, dass kein Interessenkonflikt besteht. 